\title{
Long Term Evolution Networks Planning
}

\section{Design of Program for Optimization of Next-Generation Networks with Focus on LTE}

\author{
Jan Černý, Jiří Masopust \\ Dpt. Of Applied Electronics and Telecommunications \\ FEE, University of West Bohemia \\ Pilsen, Czech Republic \\ cernyj44@students.zcu.cz, masopust@kae.zcu.cz
}

\begin{abstract}
This work deals with $4^{\text {th }}$ Generation Long Term Evolution (LTE) standard for mobile telecommunication. LTE comes with new approaches how to increase speed and stability of wireless data transmission. Planning these networks involves many complex tasks, therefore advanced software tools are needed. The mostly used are Atoll or ASSET. This simulation described in the end of this paper is attempting to demonstrate the most important principles of the planning process.
\end{abstract}

Keywords-LTE, Long Term Evolution, Radio Network Planning, Cell Site Planning

\section{INTRODUCTION}

Mobile data traffic globally multiplied fourteen times between 2010 and 2015 according to Ericsson mobility report [1]. Long-term Evolution (LTE) standard is focused on maximum data throughput to follow this trend. It is very complex system, which involves modern technologies from various fields, starting with optical technology of the backbone network followed by complex electronics for signal processing and control. Very important are the technologies applied to maximize the frequency-bandusage efficiency and increasing the data throughput.

After a brief description of the whole system of LTE the most important of the frequency-efficiencymaximizing technologies are mentioned. Afterwards the process of planning the network in general is described and the most commercially used professional software tools are named. In the end is presented a simulation program created in MATLAB to approach the network planning process.

Similar approach to this project can be found in the bachelor thesis [10], which uses the Berg's recursive model to predict coverage of microcells. Simulations of signal propagation for mobile networks in Matlab can be also found in the publication [11] and the LTE principles are closely explained in the publication [12] also using Matlab programs.

\section{CHARACTERISTICS OF LTE}

Long-term evolution (LTE) is a cellular technology standard following after the third generation UMTS networks. Therefore LTE is considered 4th generation cellular network (4G). LTE is fully IP based and focused on delivering multimedia content with improvement of Quality of Service (QoS). The most important technologies allowing LTE reach high data speed over the air interface within a limited broad band are Orthogonal Frequency Division Multiple Access (OFDMA), Multiple-Input MultipleOutput (MIMO) and Adaptive Modulation and Coding (AMC). The result is a network with maximum data throughput $300 \mathrm{Mbit} / \mathrm{s}$ for downlink and $75 \mathrm{Mbit} / \mathrm{s}$ for uplink. Very important is also reduced latency. LTE network is backwards compatible with existing technologies and supports inter-system handover both ways. [4]

\section{A. Orthogonal Frequency Division Multiple Access (OFDMA)}

Data transmission over the air interface is based on the Orthogonal Frequency Division Multiplexing (OFDM). OFDM divides frequency specter into multiple closely spaced orthogonal sub-carriers. Every single subcarrier can have much lower symbol rate than one currier would have while occupying the whole band. This efficiently eliminates the effects of multi-path. Reflected signals do not interfere with the next symbol, because all the reflected signals arrive during one symbol duration. To avoid any intersymbol interference, the guard interval is implemented after every symbol.

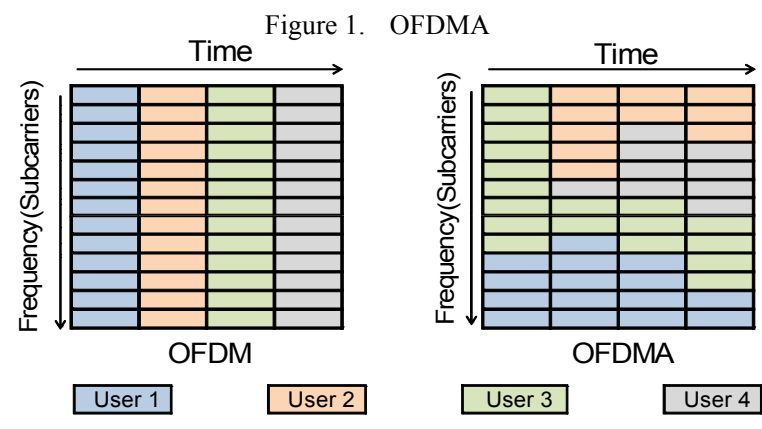

This picture shows extension of OFDM, OFDMA, OFDMA assigns carriers to users dynamically, following their current need of bandwidth.

For the purpose of a network with many subscribers, who are accessing different content or service in the same time, the subcarriers are dynamically assigned to the subscribers according to their requirements and the overall traffic within the cell. This extension of OFDM called Orthogonal Frequency Division Multiple Access (OFDMA) is used in LTE for downlink. For uplink a Single Carrier Frequency Division Multiple Access (SC-FDMA) was chosen for its better power efficiency. [2] 


\section{B. Multiple-Input Multiple-Output (MIMO)}

Theoretically, the easiest way of increasing data speed is to widen the frequency bandwidth. Unfortunately, mobile networks providers are always limited by the bandwidth. Therefore different approach of increasing the speed was needed. Multiple-Input Multiple-Output (MIMO) is one of the solutions. This name stands for the technology where multiple antennas are transmitting multiple different data streams using the same frequency. The antennas are detached, therefore each signal has a different path. This is necessary for the receiver to distinguish between the data streams. The rest is a matter of signal processing on the side of the receiver. This technology is already implemented in 3G but in LTE is improved about so-called cross-polarization. This means that each of two signal waves are polarized in a plane rotated 45 degrees from the horizontal and 90 degree to each other. Cross-polarization helps the receiver distinguish more distorted and attenuated signals. Usage of MIMO is negotiated with the eNB. When the receiver is no longer able to distinguish the data streams, MIMO is switched off or used in different mode, where it helps to increase coverage or cope with interference. [2] [3]

\section{Adaptive Modulation and Coding (AMC)}

Another factor defining the data speed is the number of bits per symbol, which is given by the used modulation. Modulations used in LTE are QPSK, 16QAM, 64QAM or 64QAM. Adaptive modulation and coding (AMC) is responsible for choosing the highest modulation possible with respect to the current channel characteristics. 64QAM modulation provides 6 bits per symbol, but the transmission becomes more sensitive to noise. Therefore, in case of worse signal conditions, a modulation with lower number of bits per symbol is chosen to keep an acceptable bit error rate. This system ensures maximum data speed in an area of good coverage and stable error-free connection in an area with weaker signal or lower Signal-toNoise-plus-Interference-Ratio (SNIR). This typically happens on the edges of a cell. [4]

\section{Network Planning [4]}

As it was mentioned before, mobile network is very complex and therefore expensive technological system. Any mistake in the process of planning the network results in increase of costs, degrades the efficiency of the whole building process and also of the network performance. The goal of network planning is to establish a radio network with sufficient coverage and capacity to ensure expected quality of service. All the resources must be used with the maximum efficiency. Therefore the particular area must be carefully studied in terms of geographical character, residential character and population density. Already existing networks must be taken in account. Well planed network should also be prepared for possible future development.

\section{A. Cell Site Planning}

Cell site planning is the whole process of finding the accurate location for the eNodesB sites and defining the size of its cells. One eNodeB typically serves three cells. Cell site planning consists of many different interconnected tasks which can be divided in surveys, coverage planning, capacity planning, frequency planning.

Figure 2. Cell Site Planning

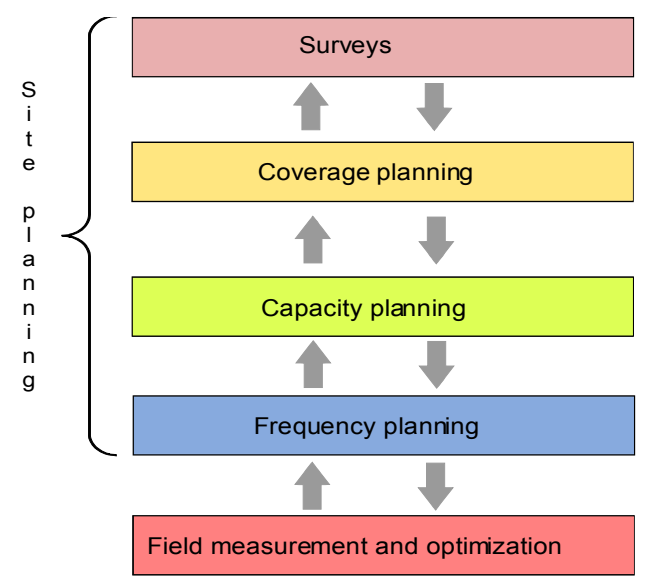

All the tasks o cell site planning are interconnected and one task cannot be carried out without taking in account the impact on the other part of the process.

\section{1) Coverage Planning}

Coverage planning serves to identify spots with low signal and adjust the position or the power of the eNodeB to efficiently cover the whole desired area. To calculate coverage, propagation models are used. Propagation models can be either empirical/statistic or deterministic. Empirical propagation models are typically based on COST-231 model. They give general results about the signal fading as a function of frequency and distance from the eNodeB. Additionally they are tuned by other additional parameters like general type of environment or relative difference of the antennas height. Deterministic models give more accurate results than empirical. They are based on determining exact ways of wave propagation path. To approach these accurate results, the simulation tool requires more specific data about the area and they are more demanding on computing resources. Threedimensional ray tracing is an example of deterministic model used in LTE planning. To reach higher accuracy with planning additional field, measurement must be carried out to verify and adjust the results of the simulation. Choice of frequency has also impact on coverage. Higher frequencies have higher path loss and therefore maximum size of a cell is smaller. [4]

\section{2) Capacity Planning}

Capacity planning of LTE networks is usually based on measured data obtained from existing network. Capacity of a cell is limited and one cell can handle only a limited amount of subscribers. Therefore in areas with higher expected traffic (for example a city center) the cell size must be smaller compared to suburban or rural areas.

Arrival of smart-phones brought completely new challenges for capacity planning. Smart-phones are constantly going from idle state to connected, when applications synchronize with a server. Smart-phones are also more demanding on data speed and therefore require more bandwidth. [4] 
To ensure maximum data speed but also to satisfy maximum users, the bandwidth is assigned dynamically (see OFDMA). With more active users in a cell the data speed for one user degrades. To deliver important real time services, as for example voice or video-call in conditions of higher cell traffic, must be implemented well defined Quality of Service protocol. With increasing traffic in the network the interference grows, which has also significant impact on capacity. Interferences can be limited with correct frequency planning. [2] [4]

\section{3) Frequency planning}

3 GPP defines various frequency bands in range from $700 \mathrm{MHz}$ to $3800 \mathrm{MHz}$ and also defines different bands for Frequency Division Duplex (FDD) and for Time Division Duplex (FDD). The used bandwidth can be $1,4 \mathrm{MHz}, 3 \mathrm{MHz}, 5 \mathrm{MHz}, 10 \mathrm{MHz}, 15 \mathrm{MHz}$ or $20 \mathrm{MHz}$. This gives the operators possibility to implement frequency band available in their area.

Instead of using different frequency carriers in adjacent cells, like for example in GSM, in LTE Conventional Frequency reuse Scheme $1 * 3 * 1$ can be used. Then the whole frequency band is assigned to each cell. That improves the capacity of each cell but results in higher interference on the borders of the cells. LTE uses scrambling and pseudo-noise codes to distinguish between signals on the same frequency. [4] Still the interferences are present and result in a noise which according to the Shannon-Hartley theorem degrades maximum possible data throughput. Therefore in urban areas with high site density it is recommended to use Soft Frequency reuse scheme $1 * 3 * 1$ (Fig. 3). In this case only part of the cell is covered by the whole frequency band. The edges of the cell are covered only by one third of frequency band, different third than the adjacent cell is using. Frequencies used for the edges are following reuse scheme known from cell networks like GSM. This helps to reduce the inter-cell interferences. [2]

Figure 3. Soft Frequency reuse scheme

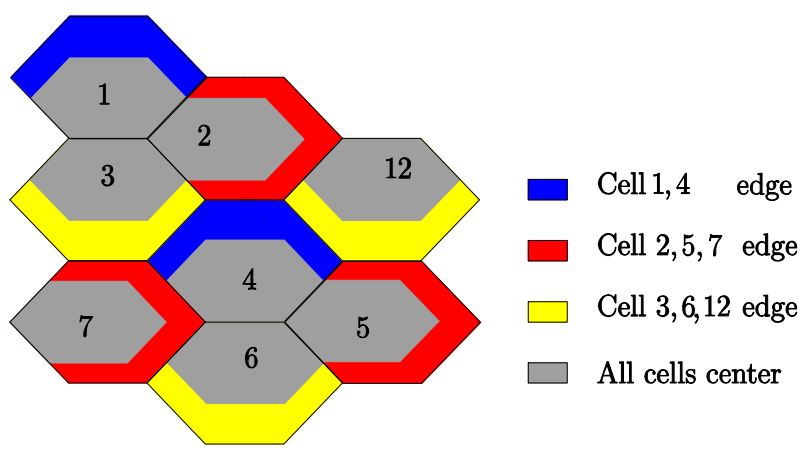

\section{B. Professional software}

To help the engineers with all the complex tasks connected with the network design there are various Radio Network Planning (RNP) software tools. Based on information about the network configuration and characteristics of the environment, the RNP tool provides graphical outputs visualizing coverage, capacity and interference in the network area.
RNP software tools must contain characteristics of used network standard and equipment to give applicable results. Therefore the proper planning software tools must be used for planning of LTE networks.

Figure 4. Function of RNP software tool

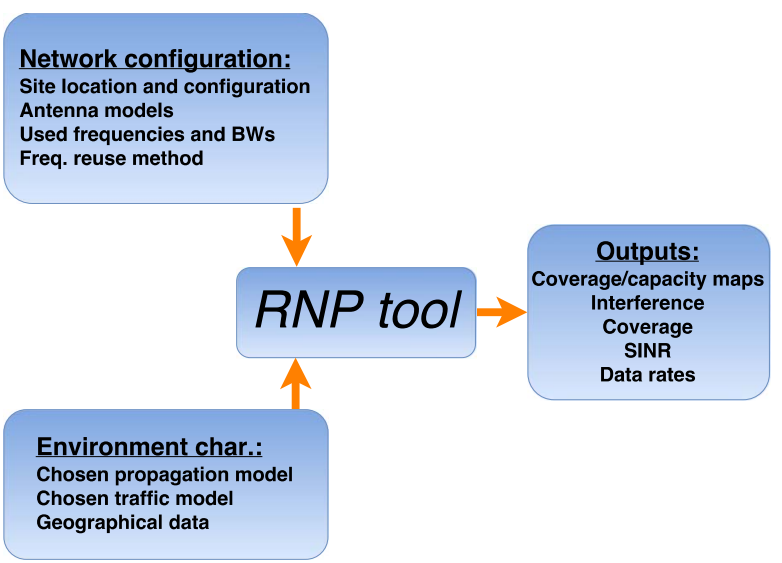

Radio Network Planning (RNP) tool calculate and visualize its outputs based on introduced data about the network configuration and the particular environment. [6]

According to [5] and [7] the most popular RNP software is Atoll. Atoll is a multi-technology wireless network design and optimization software tool suitable for many standards including GSM, UMTS and LTE. It supports multi-technology simulation suitable for planning LTE networks along with other standards. It includes various adjustable propagation models both empirical and deterministic. Atoll also supports various sources of geographical data including popular web map services. Other wide spread software tools are for example ASSET or Pegaplan [8], which is used by Czech T-mobile.

Figure 5. Example of coverage map output

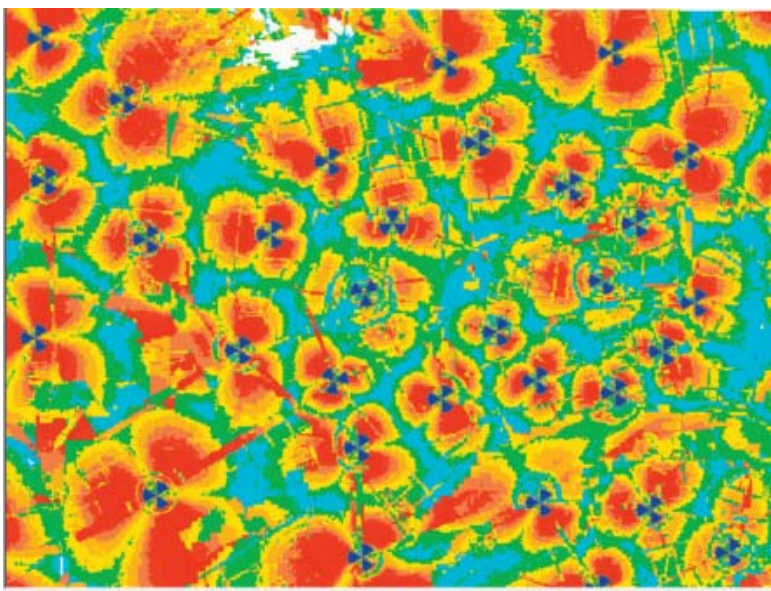

The coverage calculation gives location-dependent results. One coverage result is the maximum data rate which a user achieves at a certain location in a radio cell. This means in the context of radio network planning that this achievable data rate is calculated for every pixel. [8]

\section{DESIGN OF PROGRAM FOR OPTIMIZATION OF} LTE NETWORK

In the bachelor thesis [9] multipath signal propagation and empirical models used for planning of 2G GSM cellular networks were studied and a 
simulation program approaching cellular network planning was created in Matlab. The main objective now is to adjust the simulation so it can be applied on $4 \mathrm{G}$ networks.

The simulation has a graphical user interface allowing a user to place different eNodeBs in the simulation area, assign them frequency band and define maximum emitted power. Positions and information about the eNodeB can be also loaded from saved text file. After that the signal coverage map based on chosen propagation model is calculated. Individual sites can be changed or deleted to adjust the coverage. It is also possible to visualize zones with unacceptable interference.

Calculation of coverage is based on distance from the antenna and defined frequency band. It does not take in account antenna characteristics. However the signal spreading is restricted on a 120 degree sector to serve the cell from its border, as it is typical in both GSM and LTE networks.

Figure 6. Example of coverage map output

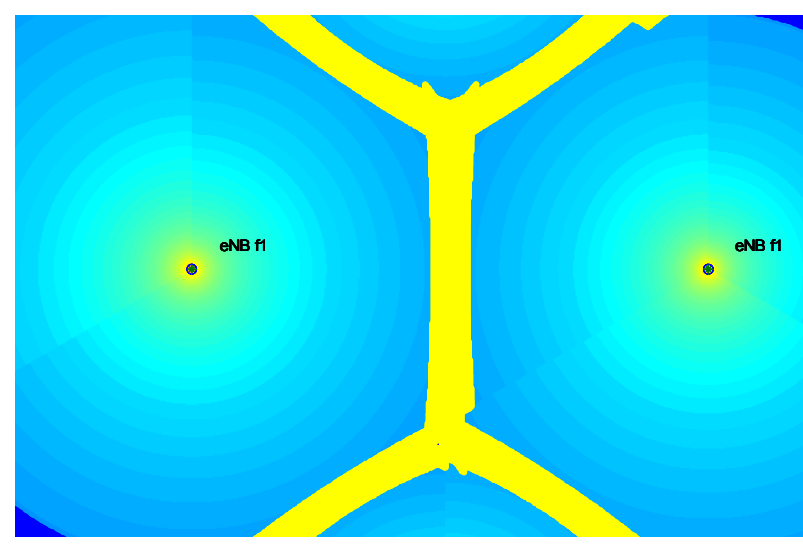

In this picture is we can see two eNodeBs serving six cells and parts of two adjacent cells. All the cells are covered with the same frequency. Coverage is calculated based on Empirical model [9]. Yellow zone symbolize area of unacceptable level of interferences for a GSM network and absence of stronger carrier signal.

The interference simulation visualizes areas of network with low Currier-to-Interference ratio. It is calculated as a difference of the currier signal level and the strongest interfering signal on the same frequency. In GSM networks it was necessary to avoid interference in order to ensure the service there. In LTE interference mainly limits the data speed. Data speed is also one of the most observed parameters at LTE. Therefore it is suitable to implement visualization of data throughput map to the simulation. It can be mainly based on existing coverage and interference calculation and it should take in account also deployment of MIMO technology and AMC.

\section{CONCLUSION}

LTE networks are very complex and their planning takes in account many factors. All of the tasks of site planning (coverage planning, capacity planning, frequency planning) are interconnected and cannot be carried out separately. Professionally used Radio Network Planning software simulates the coverage and other indicators important for planning with accuracy, which will be hard to approach with this fairly simplified simulation. However it can be very useful to visualize the basic principles and phenomena in this easy-to-use tool. In order adapt the simulation for the LTE network standard it is necessary to take in account used technologies as MIMO and AMC and provide data throughput visualization.

\section{REFERENCES}

[1] Ericsson (2015, June). Ericsson Mobility Report. Retrieved February 24, 2016, from http://www.ericsson.com/res/docs/2015/ericsson-mobilityreport-june-2015.pdf

[2] Huawei (2011). Long Term Evolution (LTE) Radio Access Network Planning Guide. Retrieved March 1, 2016, from http://www.slideshare.net/tharinduwije/lte-radio-netwokplanning

[3] Telco Antennas (n.d.). What is MIMO? Retrieved March 07, 2016, from https://www.telcoantennas.com.au/site/how-doesmimo-work

[4] Song, L., Shen, J., Stasiak, M., Zwierzykowski, P., \& Głąbowski, M. (2011). Evolved celluar network planning and optimization for UMTS and LTE. Boca Raton: CRC Press Taylor \& Francis Group

[5] Waqas Akram, M. (2012, May 14). Finding the best Radio Network Planning and Radio Network Optimizatio... Retrieved February 20, 2016, from

http://www.slideshare.net/waqasakram940/finding-the-bestrnp-and-rno-software

[6] Pietrzyk, S. (2012). LTE Radio Network Planning Tutorial. Retrieved February 18, 2016, from http://www.slideshare.net/IS-Wireless/lte-radio-networkplanning-tutorial-from-iswireless?qid $=4 \mathrm{fb} 71 \mathrm{c} 09-13 \mathrm{fe}-4 \mathrm{f} 41$ 9877-90c9d2ae52c2

[7] Atoll Overview. (n.d.). Retrieved February 21, 2016, from http://www.forsk.com/atoll/

[8] Liesenfeld, B., Lustig, M., Weller, A., Oppermann, E., \& Beyer, J. (2010). LTE-Radio Network Planning with PegaPlan. Retrieved February 20, 2016, from http://www.pegaware.com/fileadmin/PegaWeb/content/autore n/data/news/LTE_radio_network_planning_english.pdf

[9] Černý, J. (2015). Design of Program for simulation of Signal Coverage in Cell Network. University of West Bohemia.

[10] Unzeitig, L. Cellular Network Coverage Model. Brno University Of Technology, Faculty of Electrical Engineering and Communication, 2008

[11] Fontan, F. Pérez and P. Mariño Espiñeira.(2008). Modelling the Wireless Propagation Channel: A simulation approach with Matlab. ISBN: 978-0-470-75173-2

[12] Zarrinkoub, Houman. (2014). Understanding LTE with MATLAB: From Mathematical Modeling to Simulation Prototyping. Print. ISBN: 978-1-118-44341-5 